\title{
Decoding perceptual thresholds from MEG/EEG
}

\author{
Yousra Bekhti*† , Nicolas Zilber*ף, Fabian Pedregosa ${ }^{\ddagger \uparrow}$, Philippe Ciuciu ${ }^{\ddagger}$, \\ Virginie van Wassenhove* ${ }^{*}$, Alexandre Gramfort ${ }^{\dagger} \uparrow$ \\ *INSERM, U992, Neurospin bât 145, 91191 Gif-Sur-Yvette, France \\ $\dagger$ Institut Mines-Telecom, Telecom ParisTech, CNRS LTCI, Paris, France \\ $\ddagger$ Parietal Team, INRIA Saclay-Île-de-France, Saclay, France \\ $\S$ Univ Paris-Sud, Cognitive Neuroimaging Unit, F-91191 Gif/Yvette, France \\ ${ }^{\top} \mathrm{CEA}, \mathrm{DSV} / \mathrm{I}^{2} \mathrm{BM}$, NeuroSpin Center, F-91191 Gif/Yvette, France
}

\begin{abstract}
Magnetoencephalography (MEG) can map brain activity by recording the electromagnetic fields generated by the electrical currents in the brain during a perceptual or cognitive task. This technique offers a very high temporal resolution that allows noninvasive brain exploration at a millisecond $(\mathrm{ms})$ time scale. Decoding, a.k.a. brain reading, consists in predicting from neuroimaging data the subject's behavior and/or the parameters of the perceived stimuli. This is facilitated by the use of supervised learning techniques. In this work we consider the problem of decoding a target variable with ordered values. This target reflects the use of a parametric experimental design in which a parameter of the stimulus is continuously modulated during the experiment. The decoding step is performed by a Ridge regression. The evaluation metric, given the ordinal nature of the target is performed by a ranking metric. On a visual paradigm consisting of random dot kinematograms with 7 coherence levels recorded on 36 subjects we show that one can predict the perceptual thresholds of the subjects from the MEG data. Results are obtained in sensor space and for source estimates in relevant regions of interests (MT, pSTS, mSTS, VLPFC).
\end{abstract}

\section{INTRODUCTION}

Decoding or brain reading consists in predicting from neuroimaging data the subject's behavior or parameters describing the stimuli presented [1]. This approach makes use of supervised learning techniques to learn a relationship between the target variable to be predicted and the data. The performance of such models depends on the techniques employed, the level of noise, the amount of data and on the relevance of the predictive variables. The inference procedure is often particularly challenging due to the high dimensionality of data and the low number of samples available for learning. Here the samples refer to the number of observations which can be images for fMRI, trials or epochs for MEG and EEG.

There has been much work in cognitive neuroscience using decoding and MEG, due to its ability to measure fast brain responses. Several groups [2]-[4] showed that movement direction can be predicted during overt and imagined movements. Another group [5] decoded object category membership for animacy, naturalness, faces versus bodies or human versus nonhuman faces/bodies. MEG was also used to decode visual and auditory stimulus [6], [7]. Decoding methods used in those studies differ depending on what types of target labels are predicted: these can be binary targets (class A or class B) or multiclass problems. For many neuroimaging applications, 978-1-4799-4149-0/14/\$31.00 2014 IEEE the class labels are well ordered or ranked: the level of brain damage for brain diseases, pain level or the complexity of a cognitive task. Using a classifier that takes into account the ordinal nature of the target variable allows to gain statistical power compared to multi-class classification, which disregards the order information. Ranking approaches have already been employed in fMRI decoding. In [8], [9], the authors used an ordinal ranking model (a.k.a ordinal regression) to discriminate between ordered labels, and [10] introduced the use of pairwise loss functions to discriminate between pairs of fMRI images.

In this paper, we present a framework for the prediction of ordered variables using Ridge regression combined with a ranking scoring metric. We then explain how the errors of the decoder can be quantified to give some insights into the data. The method is then validated on MEG recordings acquired on 36 subjects who followed a visual paradigm for which the stimuli were parametrized by seven levels of coherent motion. We test this method on raw MEG data and on source estimates after source localization, before relating the observed results with the behavioral data.

Notation We mark vectors with bold letters, $\mathbf{a} \in \mathbb{R}^{N}$ and matrices with capital bold letters, $\mathbf{A} \in \mathbb{R}^{M \times N}$. $\mathbf{a}_{i}$ identifies the $\mathrm{i}^{\text {th }}$ vector element, $\mathbf{A}_{i, j}$ the matrix element with row index $\mathrm{i}$ and column index $\mathrm{j}$. We indicate the $\ell_{2}$ norm of a vector $\mathbf{a}$ as $\|\mathbf{a}\|_{2}=\left(\sum_{i}\left|\mathbf{a}_{i}\right|^{2}\right)^{\frac{1}{2}}$.

\section{MAterials AND Methods}

Supervised learning on neuroimaging data, a problem commonly referred to as decoding, consists in predicting a target variable $y \in\{1, \ldots, K\}$ from input data $\mathbf{x} \in \mathbb{R}^{p}$. When using a linear regression model, the target values are derived from a linear combination of the data, $\mathbf{y}=\mathbf{X w}$, where $\mathbf{w}$ is a weight vector and $\mathbf{X}$ is a $n$-by-p data matrix with $p$ features and $n$ observations. In the case of MEG/EEG, observations are the repetitions of the experiment also called trials or epochs. Here $K$ will be equal to 7 defining the number of classes/target variables. While it could be possible to use a multi-class classification approach as decoder, such as a strategy ignores the fact that the targets are ordered. For example, it is worse to predict 5 instead 2 than 3 .

The Ridge regression model is defined as the solution to the 
convex optimization problem:

$$
\hat{\mathbf{w}}=\underset{\mathbf{w}}{\arg \min }\|\mathbf{y}-\mathbf{X w}\|_{2}^{2}+\lambda\|\mathbf{w}\|_{2}^{2}
$$

and is a popular approach to predict a linear relationship between the target values and the input data. While this approach respects the order of the targets it does not offer a relevant metric to evaluate the success of a decoder with an ordered set of categories.

When using a linear regression model, the mean square error (MSE) is the natural performance metric. Yet, in high dimensional settings with limited number samples $(n \ll p)$ like here, MSE is often a poor metric. To reduce the variance of the estimated $\mathbf{w}$, high values of $\lambda$ are used causing a strong amplitude bias on the coefficients $\mathbf{w}$ and poor performance when measured using MSE.

To address this issue, we propose to exploit the information that the values in $\mathbf{y}$ can be ordered. This leads us to quantify the performance in terms of ranking, where we test the ability of the decoder to properly order samples, trials, based on the target to predict. The ranking scorer consists in comparing the real values of $\mathbf{y}$ and the predicted ones. Given two trials from the validation dataset, where $\left(y_{i}, y_{j}\right)$ denote their associated labels with $\left(y_{i} \neq y_{j}\right)$. Let $\mathcal{P}=\left\{(i, j)\right.$ s.t. $\left.y_{i} \neq y_{j}\right\}$ be the set of pairs with different labels. One quantify the prediction accuracy $A c c$ with the percentage of correct orderings for pairs of trials:

$A c c=\#\left\{(i, j) \in \mathcal{P}\right.$ s.t. $\left.\left(y_{i}-y_{j}\right)\left(y_{i}^{\text {pred }}-y_{j}^{\text {pred }}\right)>0\right\} / \# \mathcal{P}$.

For each pair of trials there is two alternative options and the chance level is therefore $50 \%$. To go beyond average accuracy, it is possible to inspect for which pair of trials the decoder makes a mistake. For this, we defined an 7-by-7 matrix accuracy $\mathbf{M}$ :

$$
\mathbf{M}_{y_{i}, y_{j}}=\frac{\#\left\{(i, j) \in \mathcal{P} \text { s.t. }\left(y_{i}-y_{j}\right)\left(y_{i}^{\text {pred }}-y_{j}^{\text {pred }}\right)>0\right\}}{\#\left\{(m, n) \in \mathcal{P},\left(y_{m}, y_{n}\right)=\left(y_{i}, y_{j}\right)\right\}} .
$$

Each $\mathbf{M}_{\mathbf{i}, \mathbf{j}}$ tells us how well we distinguish the level $i$ and level $j$. Note that the matrix is symetric as we have the same score comparing levels $i$ and $j$, or $j$ and $i$.

\section{RESULTS}

We first present the experimental design before detailing results obtained on sensor space data and then on source estimates in some relevant regions of interest.

Data: 36 healthy volunteers scanned while fixing a cross during 600 to $800 \mathrm{~ms}$, followed by the apparition of two intermixed and incoherent random-dot-kinematograms (RDK) red and green populations. After 0.3 to $0.6 \mathrm{~s}$, one of the RDKs became more coherent than the other. The coherence refers to the proportion of dots moving into the same direction. (Fig. 1) illustrates the red RDK as the most coherent. Participants were asked to indicate which of the red or green populations was most coherent irrespective to the direction of motion. This test allowed evaluating participants coherence discrimination threshold by testing seven levels of visual RDK coherence,

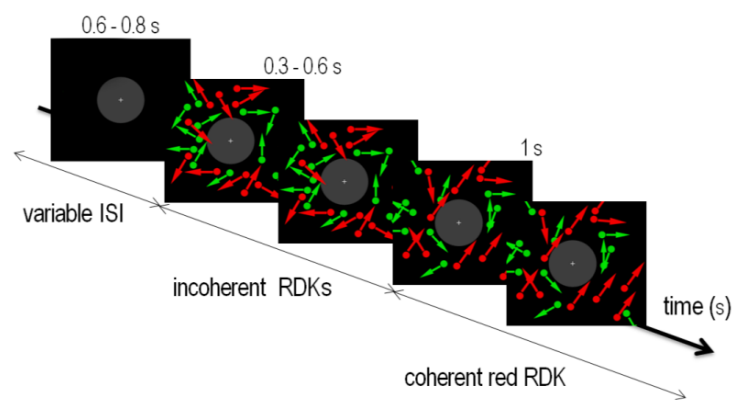

Fig. 1. Experimental trial. Participants were presented with a visual stimuli consisting in a presentation of a fixation cross, followed by the apparition of two incoherent RDKs red and green. After 0.3 to $0.6 \mathrm{~s}$, one population red or green became more coherent than the other with a certain level of coherence, 7 in all. Here $75 \%$ of red dots are moving coherently, lasting $1 \mathrm{~s}$.

namely: $15 \%, 25 \%, 35 \%, 45 \%, 55 \%, 75 \%$ and $95 \%$. A total of 196 trials were tested for each subject, 28 trials for each coherence level. The paradigm is summarized in Fig. 1.

\section{A. Sensor space}

We first report decoding results on the 7 levels of coherence using as input data the 204 sensors (only gradiometers). The decoding analysis included different steps: 1) identification of the time window: we first performed a time-by-time decoding to get a score for each time-point. The time interval containing the time points with scores slightly above chance was then used to define the time window of interest: 100 to $600 \mathrm{~ms}$ after coherence onset. 2) prediction of coherence levels using ridge regression and the ranking scorer.

In sensor space, on per subject basis decoding was performed, so across all trials of one subject. Using 204 gradiometers and a time window from $100 \mathrm{~ms}$ to $600 \mathrm{~ms}$ (126 time-points), the dimensions of the data are: $n=196$ (28 trials $\times 7$ coherence levels) at most depending on the number of dropped epochs, and $p=204 * 126 \sim 2.5 \times 10^{4}$. We evaluate the performance of the method with a 10 -fold stratified cross-validation (i.e., which preserves the percentage of sample for each class/coherence level in each fold). Fig. 2$\mathrm{a}$ is the accuracy matrix averaged over the 36 participants. We observe that the more the pairs of trials are different in terms of coherence, the easier it is to order them. Still the matrix shows two types of entries: entries close to chance level of $50 \%$ ( 0.5 score), and significant entries above $60 \%$ accuracy. This suggests the presence of two brain states. For the 4 coherence levels below 55\%, the MEG signals do not allow to discriminate the levels. It is visible with the 4 by 4 white square matrix in the upper left with values around $50 \%$ accuracy. A 2 by 2 matrix in the lower right, shows that the $\mathrm{M} / \mathrm{EEG}$ data do not allow to disambiguate the 2 highest coherence levels. The natural question to ask is: Does this threshold at $55 \%$ coherence reflect subjects' behavior?

We recorded during the experiment the subjects' responses which reported which color of dots was the most coherent. We fitted a psychometric curve with a Weibull function in order to extract each individual's discrimination threshold. In Fig. 2-b, 
we averaged the psychometric curves of all subjects. We report in dashed line the perceptual threshold which corresponds to a correct response rate of $75 \%$. We note the agreement between the behavioral threshold and the threshold extracted only from the matrix $\mathbf{M}$ obtained from the MEG data.

In the second step, to facilitate the visualization and the comparaison of the matrix and the perceptual threshold, the ranking scores were converted to p-values assuming each prediction is drawn from a Bernouilli distribution with appropriate parameter. As each prediction compares two coherence level, as one is higher or lower than the second, then a chance level of $50 \%$. Thus, the parameter of the distribution is $p=0.5$. We derived a $\mathrm{p}$-value for a $i$ th coherence level from the Binomial distribution, as we average multiple Bernouilli variables. First, we computed how many times we ordered correctly all the levels higher and lower than $i$ (the same way the perceptual threshold is defined). This corresponds to a success rate that allows to derive a p-value after counting the total number of pairs.

We report in Fig 2-c, the negative mean of the logarithm to base 10 of the p-values across subjects (See Fisher's method for combining independent tests). This procedure is not meant to yield valid p-values, but to compare decoding performance across the different thresholds. The maximum of this quantity confirms a threshold in the MEG data around $55 \%$.

\section{B. Source space}

Decoding analysis showed that a significant amount of information can be extracted from MEG sensor signals used to predict coherence levels. From a neuroscience perspective, it is worth further inverstigating neuroanatomical organization, and understanding which brain regions make it possible to decode the coherence levels from sensors data. For this, we trained our decoder on 5 cortical regions of interest (ROIs) separately after source localization using dSPM [11] and the MNE software [12]. The time window from 100 to $600 \mathrm{~ms}$ was used (126 time-points). The ROIs were delineated on each participant (for more detail [13]) for both right and left hemispheres (Fig.3), except the frontalpole region which is a label from Freesurfer parcellation [14]. The dimensions of the data became: $n=196$ at most, and $p=126 * 9000 \sim 10^{6}$ at most depending on the size of the label. Using the same evaluation used for sensors data, we investigated the decoding within hMT+, a region known to be involved in the processing of coherent visual motion. Smaller patterns can be seen in the accuracy matrix of Fig. 4, and performance is slightly lower. This can be explained by the restriction to a small ROI while sensors data are sensitive to the whole brain. It suggests that $\mathrm{hMT}+$ is not the only region implicated in the discrimination of the coherence levels. Still, we observe the same transition in the matrix $\mathbf{M}$ with a threshold of $55 \%$.

In Fig. 5) we report the performance for different ROIs. The coherence level corresponding to the lowest p-value represents the level for which we best discriminate pairs of trials, one higher and one lower than this level. Overall, and as predicted , hMT+ yielded the best discriminative power.

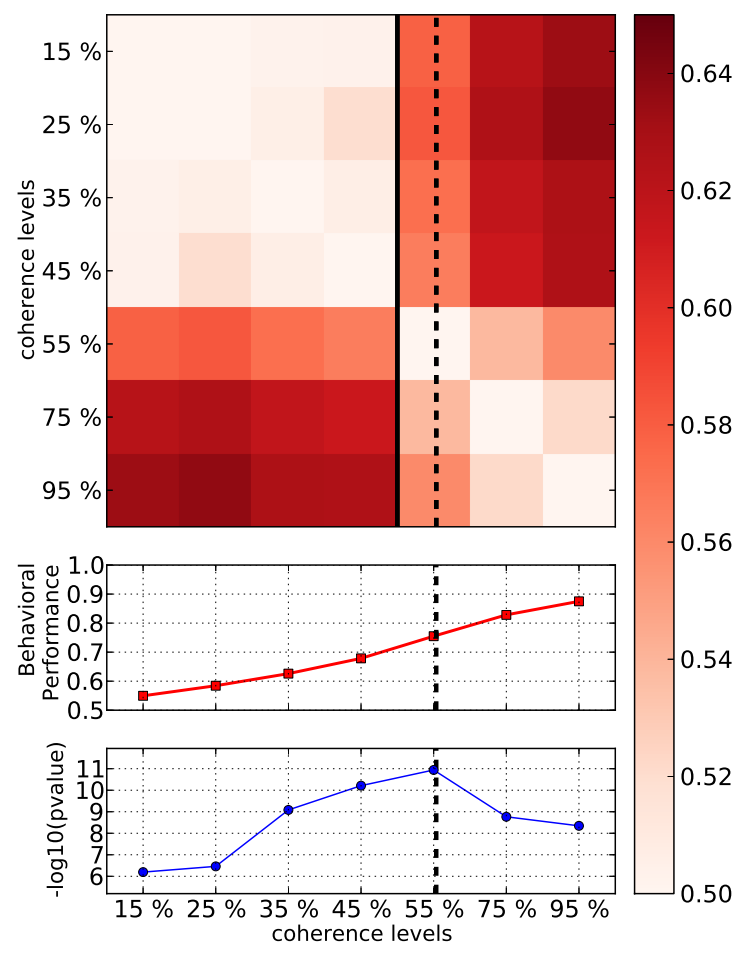

Fig. 2. (a) Mean of accuracy matrix across all subjects was computed from sensors data. Accuracy Matrix starts from chance level $0.5(50 \%)$ and shows how well we distinguish the different coherence levels. (b) Mean performance across all participants as a function of coherence levels. Black dashes corresponds to the perceptual threshold averaged over all subjects. Black line matches the transition between the two patterns: enough distinguishable levels from not enough. (c) Mean of $-\log 10$ of p-values on each coherence level across subjects.

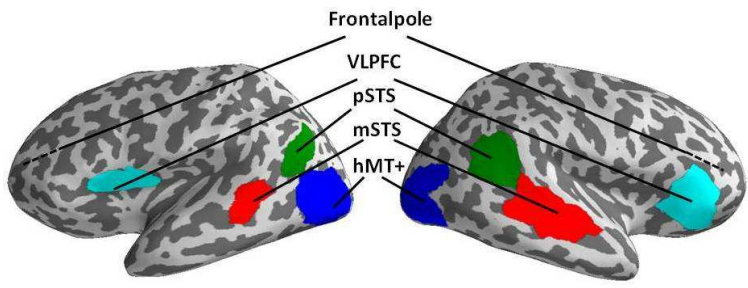

Fig. 3. Regions of interest (ROIs) delineated on one participant. pSTS: posterior Superior Temporal Sulcus. mSTS: middle Superior Temporal Sulcus. VLPFC: VentroLateral PreFrontal Cortex.

Small differences were noticed comparing the p-values of the two lowest levels ( $15 \%$ and $25 \%$ ), then the p-values of the two highest levels $(75 \%$ and $95 \%)$ for all labels. This defined the hardest $(15 \%$ and $25 \%)$ and the easiest $(75 \%$ and $95 \%$ ) levels to detect. This small difference is explained by the almost same encoding for those two-by-two extreme levels. While large differences between the p-values of the levels in the middle are perceived. The higher the level, the lower the p-value until reaching the peak of the perceptual threshold. Of all regions of interest, vLPFC was the label of least discriminative power albeit it perserved discrimination in the region of highest learning during the task - i.e. around the perceptual threshold. pSTS showed a lower p-value than 

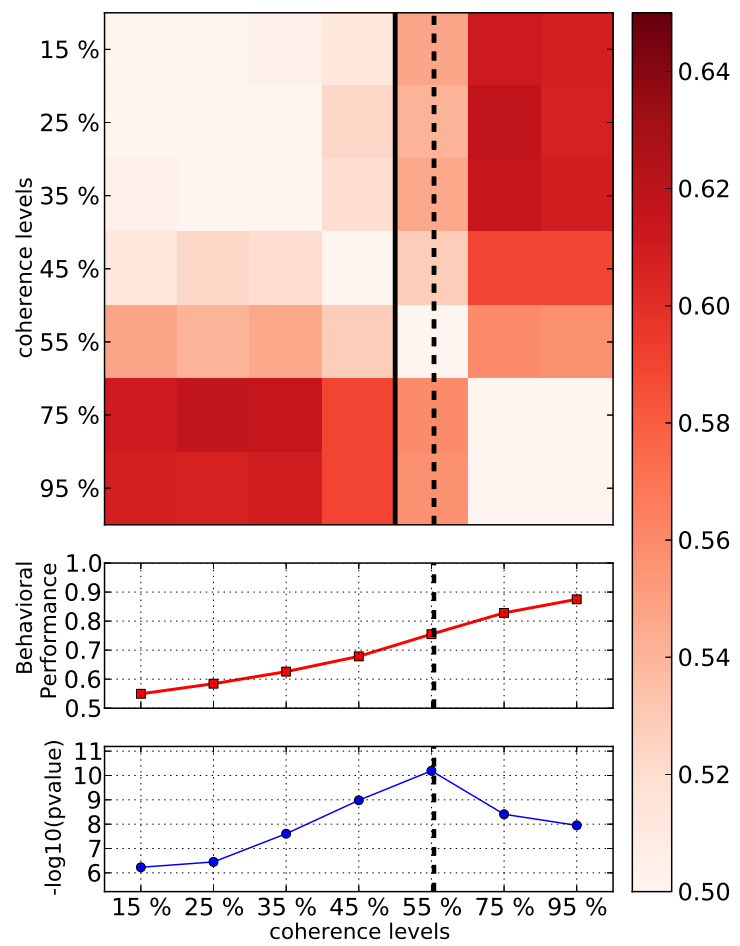

0.52 0.50

Fig. 4. (a) Mean of accuracy matrix across all subjects was computed from hMT+ label. Matrix of accuracy starts from chance level 0.5 (50\%) and shows how well we distinguish the different coherence levels. (b) Mean of behaviors accross all participants as a function of coherence levels. Black dashed line corresponds to the perceptual threshold averaged over all subjects, Black line matches the transition between the two patterns : enough distinguishable levels from not enough. (c) Mean of $-\log 10$ of pvalues on each coherence level across subjects.

hMT + but displayed a faster curve increase. This could be accounted by the multisensory integrative role of pSTS during learning. Similarly, mSTS showed a better discriminative power below the perceptual threshold. The regions of interest defined on basis of prior analysis [13] nicely show specificity for decoding. On the contrary, a control label (here, the frontal pole, black curve) barely reflects the perceptual threshold.

\section{Discussion AND CONCLUSION}

In this paper, we proposed a data-driven procedure to detect perceptual thresholds using MEG data. We proposed an innovative approach to measure decoder's performance when working with ordered targets and demonstrated how the predictions errors can offer interesting insights on the data. Rather than using a multi-class classifier blind to targets order and with little training samples per class, we used a ridge regression with a pairwise ranking scorer. Altogether, our results suggest that decoding brain activity in a visual task may enable to reliably derive participants' perceptual threshold changes. Additionally, decoding results in source space bring out reliable discriminative power across regions known to be implicated in the task. Future work will take into consideration additional dynamic aspects of the MEG signals, and test the

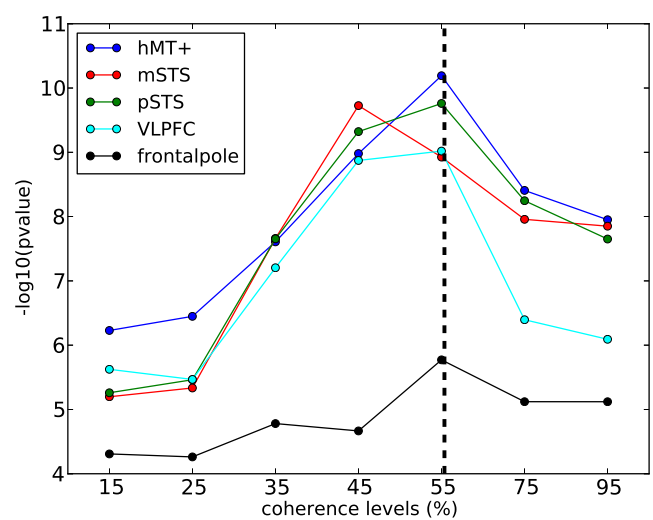

Fig. 5. - $\log 10$ of pvalues obtained from accuracy matrices of 5 ROIs as function of level of coherence. Black dashes corresponds to the perceptual threshold averaged over all subjects.

discriminative power of decoding techniques as a function of the networks implicated in the task.

\section{REFERENCES}

[1] R. A. Poldrack, "Can cognitive processes be inferred from neuroimaging data?" Trends in Cognitive Sciences, vol. 10, no. 2, pp. 59 - 63, 2006.

[2] A. Georgopoulos, F. Langheim, A. Leuthold, and A. Merkle, "Magnetoencephalographic signals predict movement trajectory in space," Experimental Brain Research, vol. 167, no. 1, pp. 132-135, 2005.

[3] S. Waldert, H. Preissl, E. Demandt, C. Braun, N. Birbaumer, A. Aertsen, and C. Mehring, "Hand movement direction decoded from MEG and EEG," Journal of Neuroscience, vol. 28, no. 4, pp. 1000-1008, 2008.

[4] W. Wang, G. P. Sudre, Y. Xu, R. E. Kass, J. L. Collinger, A. D. Degenhart, A. I. Bagic, and D. J. Weber, "Decoding and cortical source localization for intended movement direction with MEG," Journal of Neurophysiology, vol. 104, no. 5, pp. 2451-2461, 2010.

[5] R. M. Cichy, D. Pantazis, and A. Oliva, "Resolving human object recognition in space and time." Nature Neuroscience, 2014.

[6] P. Ramkumar, M. Jas, S. Pannasch, R. Hari, and L. Parkkonen, "Featurespecific information processing precedes concerted activation in human visual cortex," The Journal of Neuroscience, vol. 33, no. 18, pp. 76917699, 2013.

[7] J.-R. King, A. Gramfort, A. Schurger, L. Naccache, and S. Dehaene, "Two distinct dynamic modes subtend the detection of unexpected sounds," PLoS ONE, vol. 9, no. 1, p. e85791, 012014.

[8] Y. Fan, "Ordinal Ranking for Detecting Mild Cognitive Impairment and Alzheimers Disease Based on Multimodal Neuroimages and CSF Biomarkers," in Multimodal Brain Image Analysis, ser. LNCS. Springer, 2011, vol. 7012, pp. 44-51.

[9] O. M. Doyle, J. Ashburner, F. O. Zelaya, S. C. R. Williams, M. A. Mehta, and A. F. Marquand, "Multivariate decoding of brain images using ordinal regression." NeuroImage, May 2013.

[10] F. Pedregosa, A. Gramfort, G. Varoquaux, B. Thirion, C. Pallier, and E. Cauvet, "Improved brain pattern recovery through ranking approaches," in PRNI 2012 : 2nd International Workshop on Pattern Recognition in NeuroImaging, London, Royaume-Uni, Jul. 2012.

[11] A. Dale, A. Liu, B. Fischl, and R. Buckner, "Dynamic statistical parametric mapping: combining fMRI and MEG for high-resolution imaging of cortical activity," Neuron, vol. 26, pp. 55-67, 2000.

[12] A. Gramfort, M. Luessi, E. Larson, D. Engemann, D. Strohmeier, C. Brodbeck, L. Parkkonen, and M. Hämäläinen, "MNE software for processing MEG and EEG data," NeuroImage, vol. 86, no. 0, pp. $446-$ 460, 2014.

[13] N. Zilber, P. Ciuciu, A. Gramfort, L. Azizi, and V. van Wassenhove, "Supramodal processing optimizes visual perceptual learning and plasticity," NeuroImage, 2014.

[14] B. Fischl, "FreeSurfer," NeuroImage, vol. 62, no. 2, pp. 774-781, Aug. 2012. 